\title{
Structural Design and Modeling Analysis of Carbon-free Car
}

\author{
Yiming Xiao ${ }^{\mathrm{a}}$ \\ School of Energy, Power and Mechanical Engineering, NCEPU, Baoding 071003, China \\ a1661388871@qq.com
}

Keywords: Kinematics, Differential equations, Structural design.

\begin{abstract}
This paper presented a new carbon-free car design, and analyzed its motion. We established a physical model and established differential equations based on kinematic equations. We used the Mat lab software to depict the trajectory of the car. The results showed that the new structure can better optimize the carbon-free car's movement.
\end{abstract}

\section{Introduction}

Since the carbon-free car project has launched, it has gradually become good practice project to train students' thought and innovative ability. This paper puts forward a new kind of carbon-free car design; it provides new thought for the design and development of the carbon-free car.

\section{The design task}

A car is designed, according to the principle of conservation of energy, the energy converted by the given gravity potential energy provides driving, walking and turning. Given gravity potential energy is $4 \mathrm{~J}$, by uniformly using weight lead with $1 \mathrm{~kg}$ vertically drop to obtain, the drop is $400 \pm 2 \mathrm{~mm}$, after weight dropping, it must be loaded by the car and together move, it are not allowed to fall from the car. [1].

\section{The structural introduction}

The car is three-wheel structure, rear wheel drive, front wheel steering, the three wheels are distributed in isosceles triangle, car body erects a tower of truss structure in order to obtain the weight dropping height.

Weight connects thread around the top roller of tower winding on the gear shaft, by adopting first increasing speed to turn rear axle rotate, the car has two rear wheels, one is active, and the other is driven move, so as to drive the car forward.

When the car starts, it is in order to obtain larger traction, the gear shaft with a conical sleeve, thin wire winds on the conical sleeve in order to realize the accelerated launch.

After the main shaft is installed two small wheels with aprons, using friction drive disc rotate, disc shaft is vertical to rear main shaft, the lower installs crank rocker. Rocker through linear bearing installed on the front wheel spindle, control front wheel swing in cycle. [2]

Adjust the relative position between two friction wheel and friction disc, by controlling the position of the two friction wheel on the friction plate can achieve CVT, and make the car adapt the field of different roughness. To adjust movement cycle; change the length of the pendulum rod to adjust the swing; By precisely adjusting the length of the connecting rod to ensure the tracks center of car as a straight line. 


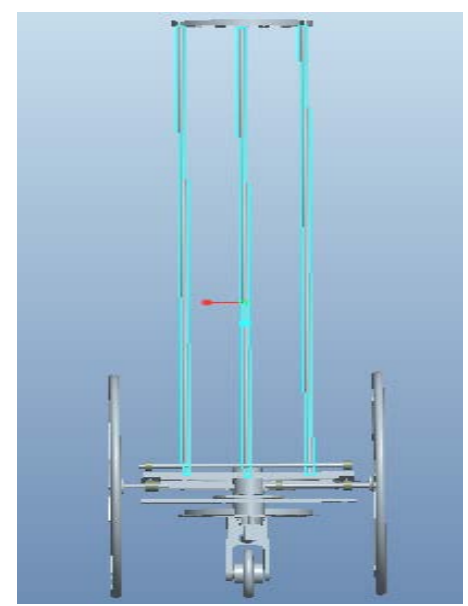

Fig.1 The design drawing of carbon-free car

\section{The model assumption}

In order to realize car as distant as possible, car wheel is as big as possible, and the friction should be as small as possible. Through the grease and other substances will be achieved. Combined with reality, the following hypotheses are put forward:

(1) Ignore the internal friction of car.

(2) Suppose the car model design as follows.

(3) Assume drive shaft and the drive wheels keep synchronized.

(4) Assume all parts have been normal.

\section{The illustration of symbols}

In this paper, the symbols involve calculation as follows:

Driving wheel radius ${ }^{\prime}$; Gear drive ratio $q$; Driving wheel A and steering wheel horizontal distance $w_{1}$; Driving wheel and steering wheel B horizontal distance $w_{2}$; The drive shaft 2 and the distance of the steering wheel $d_{1}$; 1 and the distance of the crank shaft and steering wheel $d_{2}$; the crank radius $r_{1}$; Rocker length $d_{3}$; Connecting rod length $d_{4}$; Line shaft radius $r_{2}$; The weight dropping height $h$; The rotary angle of crank shaft $2 \gamma_{1}$; rotary angle of crank shaft $1 \gamma_{2}$; The car onward distance; angle between the steering shaft and drive shaft $\alpha$.

\section{The model establishment and solution}

\subsection{The establishment of drive model}

It is relatively easy to imagine choosing drive part, it is on the top of the car off the ground $h$ whereabouts drop a weight, the weight by the rope holding, and the below connect the drive shaft, then weight pulls down the rope, the rope pulls driven rod, the rear wheel connected the driving rod will move, thus realize changing gravity potential energy of the weight into energy of wheels of the car, implements the car movement. Thus, it becomes discuss the relationship between dropping height $h$ and the drive shaft rotation, and because velocity of the drive shaft and the wheel angle is the same, the movement distance of the wheel can be concluded, then calculation of the driving part can be smoothly realized.

\subsection{The establishment of the model}

It is easy to imagine that when the car move, the rear is consistent with and front wheel, front wheel of the car rotate will inevitably lead to direction change of the rear wheel, namely there is a certain relationship between the front wheel rotation angle and the rear wheel rotation angle, the two relations determines the direction of trajectory in the moving process of the car. 


\subsection{The establishment of trajectory model}

The moving trajectory of car, it mainly can be divided into the response of the car in front of obstacles and go around obstacles, combined with both, the whole trajectory can be obtained.

\section{Solution of the model}

\subsection{Solution of drive model}

When weight dropping $d h$, the turning angle drive shaft 2 is $\gamma_{1}$, there are

$d \gamma_{1}=\frac{d h}{r_{2}}$

Then turning angle of the crank shaft

$d \gamma_{2}=\frac{d \gamma_{1}}{q}+\frac{\pi}{2}$

The car moving path length

$d s=R \cdot d \gamma_{1}$

\subsection{Solution of the turning model}

When rotating rods drive the front wheel axis deflect $\alpha$, turning point of crank shaft 1 , and $\alpha$ and $\gamma_{2}$ meet the he following spatial geometric relationship [3]:

$d_{3}{ }^{2} \cdot(1-\cos \alpha)^{2}+\left(d_{2}+d_{3} \cdot \sin \alpha-r_{1} \cdot \sin \gamma_{2}\right)^{2}-d_{4}{ }^{2}+r_{1}{ }^{2} \cdot \cos ^{2} \gamma_{2}=0$

By solving above equation, function relation between $\gamma_{2}$ and $\alpha$ is available:

$\alpha=\arcsin \frac{d_{4}^{2}-2 d_{3}^{2}-r_{1}^{2} \cdot \cos \gamma_{2}-\left(d_{2}-r_{1} \sin \gamma_{2}\right)^{2}}{\sqrt{\left[2 \cdot d_{3} \cdot\left(d_{2}-r_{1} \sin \gamma_{2}\right)\right]^{2}+\left(-2 \cdot d_{3}^{2}\right)^{2}}}$

\subsection{Solution of car moving trajectory model}

In structure model of our designed carbon-free car, A wheel as the only driving wheel, when turning angle of steering wheel is $\alpha$, when A car moving ds distance, the turning angle of car is

$$
d \beta=\frac{d s}{\frac{d_{2}}{\tan \alpha}+w_{1}}
$$

Thus we can write motion equation of the car:

$$
\left\{\begin{array}{l}
d x=d s \cdot \sin \beta \\
d y=-d s \cdot \cos \beta
\end{array}\right.
$$

\subsection{Solution of overall trajectory model of car}

In initial time rear two point cable is $\mathrm{Y}$ axis, obstacles cable is $\mathrm{X}$ axis. The coordinates of car in the moving process as follows:

$$
\left\{\begin{array}{l}
x_{B}=x-\left(w_{1}+w_{2}\right) \cdot \cos \beta \\
y_{B}=y-\left(w_{1}+w_{2}\right) \cdot \sin \beta \\
x_{C}=x-w_{1} \cdot \cos \beta-d_{1} \cdot \sin \beta \\
y_{C}=y-w_{1} \cdot \sin \beta+d_{1} \cdot \cos \beta
\end{array}\right.
$$

The path curve is drawed through Matlab as below:

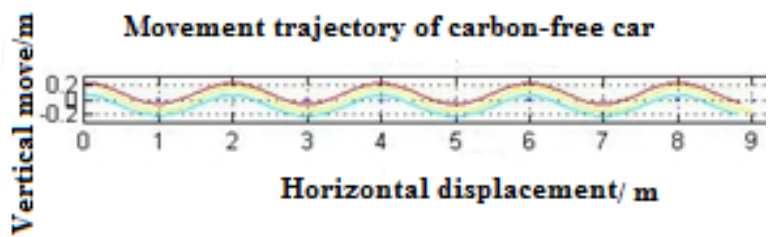

Fig.2 Movement trajectory of carbon-free car 


\section{Conclusion}

This paper carried out a series of discussion on carbon-free car driving, it puts forward a new type of carbon-free car design scheme, and the physical model was established, the kinematics principle is used to analyze, then differential equation is established to solve based on the kinematics equations, at last, Matlab software is used to design program to describe the movement trajectory of the car. Results show that this new type of structure can well optimize movement of carbon-free car.

\section{References}

[1] Zhang S W, Huang J T, Lin X R, et al. Design of "Non-carbon Small Car” [J]. Journal of Car Engineers, 2013, 06: p.33-35+54. (In Chinese)

[2] Hu H Y, Yu J P, Bao E. Structure Design and Analysis of Carbon - free Cars[J]. Journal of Dalian Nationalities University, 2013, 05: p.500-504. (In Chinese)

[3] Jiang Q Y, Mathematical Model, Higher Education Press, 2009, 11. (In Chinese) 